\title{
Semantic Web Image Search through Manual Annotation
}

\author{
M. Gokul Prasad \\ Department of Software \\ Systems \\ Karpagam University \\ Coimbatore
}

\author{
T. Sumathi \\ Department of Software \\ Systems \\ Karpagam University \\ Coimbatore
}

\author{
Dr. M. Hemalatha \\ Department of software \\ systems \\ Karpagam University \\ Coimbatore
}

\begin{abstract}
Over years the searches made over web still remains non semantic. The internet media is filled with lot of unstructured data that results this state of non semantic search over web. To make the search more specific over the query given, its attributes are to be defined while the search along with its object name. By defining so the normal web search appears to be semantic. The attributes relevant to the context is made easy over text, audio and video files. Whereas Image files still suffers with its undefined attributes along with the relevant image being uploaded. Moreover the images over web aren't tagged yet with relevant attributes in them. JPEG, Bitmap and all other commonly used image supporters yet remain without tags. Only this leads a non semantic search over images on web.
\end{abstract}

\section{Keywords}

Semantic Web, image repository, tag attributes, Resource Description Framework (RDF), Web Ontology Language (OWL), manual annotation, automatic annotation, and Meta data.

\section{INTRODUCTION}

According to Tim Berners-Lee "The Semantic Web is an extension of the current Web in which information is given welldefined meaning, better enabling computers and people to work in cooperation" $[2,27]$.

"The Semantic Web is a vision: the idea of having data on the Web defined and linked in such a way that it can be used by machines not just for display purposes, but for automation, integration and reuse of data across various applications"[3] Semantic means adding meaning of data to be discovered by computers. It is a vision of a new architecture for the World Wide Web, characterized by the Association of machineaccessible formal semantics with more traditional Web Content. The core idea is to create the meta data describing the data, which will enable computers to process the meaning of things. The ultimate goal of the Semantic Web is to transform the Web into a Medium, through which data can be shared, understood and processed by automated tools.

Semantic Web techniques, which consist of applying knowledge representation techniques in a distributed environment (potentially on a Web wide scale), have proven useful in providing richer descriptions of Web resources [4].

The Semantic Web extends the Web through the use of standards, markup languages and related processing tools. Semantic Web technologies enable people to create data stores on the Web, build vocabularies, and write rules for handling data. Linked data are empowered by technologies such as RDF and OWL.

The vision of the Semantic Web is an extension of the existing Web through which machines are able to interoperate and work on our behalf. It promises to infuse the Internet with a combination of metadata, structure, and various technologies so that machines can derive meaning from information, make more intelligent choices, and complete tasks with reduced human intervention. Semantic web vision is oriented toward machinereadable resources rather than human-readable. It requires resource description so that machines know what they mean (metadata) [5].

Currently, the World Wide Web consists of documents written in HTML. This makes the Web readable for humans, but since HTML has limited ability to classify the blocks of text apart from the roles they play, the Web in its current form is very hard to understand. The purpose of the Semantic Web is to add a layer of descriptive technologies to Web pages so that become readable. The Semantic Web is implemented in the layers of Web technologies and standards.

\section{SEMANTIC ANNOTATION IN IMAGES}

In general, manual annotation can provide image descriptions at the right level of abstraction. It is, however, time consuming and thus expensive. In addition, it proves to be highly subjective: different human annotators tend to "see" different things in the same image. On the other hand, annotation based on automatic feature extraction is relatively fast and cheap, and can be more systematic.

In the process of image annotation, we may find values for attributes or relationships that are not previously present in the knowledge base. The process of enhancing the existing metadata could be as simple as entering values for attributes; in that case they could be automated. Moreover this process could be as complex as modifying the underlying schema, in that case some user involvement might be required [11].

To annotate the images, RDF data model can be used. It is based upon the idea of making statements about Web resources in the form of subject-predicate-object expressions, called triples in RDF terminology. This mechanism for describing resources is a major component in what is proposed by the W3C's Semantic Web activity: an evolutionary stage of the World Wide Web in which automated software can store, exchange, and use machine-readable information distributed throughout the Web, in turn enabling users to deal with the information with greater efficiency and certainty. RDF's simple data model and ability to 
model disparate, abstract concepts has also led to its increasing use in knowledge management applications unrelated to Semantic Web activity [13].

To extract text content information by image pattern recognition technology would be a cost way to get semantic information of one picture as it requires complicated and optimized algorithm to realize it. To solve these challenges, RDF documents can be used as one of the alternates for image content storage because: RDF supports self-defined tags that allow user to setup new defined semantic description tags and make further modification. RDF as a mechanism for resource description would contribute to the semantic inference based on the ontology theory [29]. Its resource/property/value triples character helps to make inference based on the description documents. For example, any image can be described in several aspects like brand, shape and color.

\section{DRAWBACKS OVER RDF, OWL}

Even though RDF Editors and OWL Editors are used for annotation of attributes with the images over web, still the images over the web and the search over it remains to be meaningless. It is not quiet easy to handle these RDF and OWL editors for the end users. These Editors mainly supports some specific web languages and not all the web languages. The end user requires a greater knowledge regarding the syntax and coding of these editors since they are syntax dependent. Moreover since the coding are present for annotation the user gets annoyed to specify the attributes for the picture being uploaded. Thus they avoid tagging attributes over their images while upload, this result in unstructured data over web. These data over internet remain as garbage over internet that affects the semantic search a lot. To rectify this problem RDF editors and OWL editors were proposed yet these editors fails to structuralize the images over web. This remains to be the major drawback over semantic search. On the whole the editor's inefficiency upsets the semantic web image search.

\section{PROPOSED ALGORITHM}

The proposed algorithm will overcome the demerits of the known existing system. Here the modules are split into two i.e. Image Upload Process, Image Search Process.

\section{IMAGE UPLOAD}

\section{Step1:}

Select the file to be uploaded from the local.

\section{Step2:}

Declare appropriate object name for the image being uploaded.

Object name is primary.

\section{Step3:}

Describe the attributes relevant towards the image being uploaded. No specific constraints are specified over the attributes being specified or unspecified.

Step4:

Annotate or Tag the attributes specified with the image being uploaded in the database manually.

\section{IMAGE SEARCH}

Step1:

Get the input query from the end user,

Step2:

Perform primary search (Object Search) over the database using the first word of the given query as Object name.

Object name is primary.

Step3:

Using Logical AND operator, match the rest of the query with the attributes of the primarily searched objects.

Step4:

The images over object search are filtered and the images satisfying the end user query are produced as result.

Step5:

If no such attribute satisfying images are present in database the result will be "NOT IN DATABASE", even though if its object name is matched.

Step6:

Attributes could be null but cannot be wrong.

\section{ILLUSTRATIVE EXAMPLE}

Here by is provided with an example that clearly pictures the proposed system.

The process starts with Image Upload process.

\begin{tabular}{|l|l|l|l|l|}
\hline Image & $\begin{array}{l}\text { Object } \\
\text { Name }\end{array}$ & Color & Type & Description \\
\hline Image & Honda & Red & City & $\begin{array}{l}\text { Enjoy your } \\
\text { challenges }\end{array}$ \\
\hline Image & Honda & Black & Civic & $\begin{array}{l}\text { Pure } \\
\text { exhilaration }\end{array}$ \\
\hline Image & Maruthi & Black & SX4 & Men are Back \\
\hline Image & Honda & White & Accord & Lead \\
\hline Image & Samsung & Yellow & Corby & Next is What? \\
\hline Image & Samsung & White & Corby & Next is What? \\
\hline Image & Samsung & Black & Monte & Keep Touch \\
\hline
\end{tabular}

Here the relevant images along with the object name are being specified.

The object name here is assigned with not null constraint. 
No special editors are employed for annotation or tagging of the attributes over the image while upload.

\section{Image Search Process:}

Here end user is requested to provide the query over which his semantic search of images is being performed.

The end user has to specify the object name as the first word of the query and followed by the attributes specification.

\section{i.e., QUERY "Honda City Red"}

The splitting of words is as "Honda+City+Red+". First the primary search is carried out based on the first word "Honda", thus the images relevant with the object name Honda are collected from the database and stored in temporary array [22].

Temp array1.

\begin{tabular}{|l|l|l|l|l|}
\hline Image & $\begin{array}{l}\text { Object } \\
\text { Name }\end{array}$ & Color & Type & Description \\
\hline Image & Honda & Red & City & $\begin{array}{l}\text { Enjoy your } \\
\text { challenges }\end{array}$ \\
\hline Image & Honda & Black & Civic & Pure exhilaration \\
\hline Image & Honda & White & Accord & Lead \\
\hline
\end{tabular}

The next search is carried out with the query as "City" and "Red". Over the attributes of the images stored in the temporary array the matching is carried out, here Logical AND Operator is employed to avoid duplication.

Result array2.

\begin{tabular}{|l|l|l|l|l|}
\hline Image & $\begin{array}{l}\text { Object } \\
\text { Name }\end{array}$ & Color & Type & Description \\
\hline Image & Honda & Red & City & $\begin{array}{l}\text { Enjoy your } \\
\text { challenges }\end{array}$ \\
\hline
\end{tabular}

The resultant is stored in a secondary array which is product as result over the query of the end user. Thus resulting semantic image search. The end user describing the object name only, the search is terminated after the primary search itself. Only by specifying the attributes the search is made semantic.

QUERY “Samsung Corby”Temp array1.

\begin{tabular}{|l|l|l|l|l|}
\hline Image & $\begin{array}{l}\text { Object } \\
\text { Name }\end{array}$ & Color & Type & Description \\
\hline Image & Samsung & Yellow & Corby & $\begin{array}{l}\text { Next is } \\
\text { What? }\end{array}$ \\
\hline Image & Samsung & White & Corby & Next is \\
\hline
\end{tabular}

\begin{tabular}{|l|l|l|l|l|}
\hline & & & & What? \\
\hline Image & Samsung & Black & Monte & $\begin{array}{l}\text { Next is } \\
\text { What? }\end{array}$ \\
\hline
\end{tabular}

Result array2.

\begin{tabular}{|l|l|l|l|l|}
\hline Image & $\begin{array}{l}\text { Object } \\
\text { Name }\end{array}$ & Color & Type & Description \\
\hline Image & Samsung & Yellow & Corby & $\begin{array}{l}\text { Next is } \\
\text { What? }\end{array}$ \\
\hline Image & Samsung & White & Corby & $\begin{array}{l}\text { Next is } \\
\text { What? }\end{array}$ \\
\hline
\end{tabular}

\section{QUERY "Samsung Corby White"}

Same temp array as for the query "Samsung Corby" since the object name is "Samsung" itself

Result array2.

\begin{tabular}{|l|l|l|l|l|}
\hline Image & $\begin{array}{l}\text { Object } \\
\text { Name }\end{array}$ & Color & Type & Description \\
\hline Image & Samsung & White & Corby & $\begin{array}{l}\text { Next is } \\
\text { What? }\end{array}$ \\
\hline
\end{tabular}

Thus by specifying attributes more the search is made specific and semantic result is produced.

\section{CONCLUSION}

In this paper, I have presented a simple algorithm and logical operation to be performed over image search to make the search semantic and to avoid duplicates over the query search performed. The proposed system is a basic edition of image search engine that performs semantic image search over the user defined database. Here by I have defined only the attributes as 'color', 'type' and 'description'. More attribute defining will enhance the semantic search over images. In this basic edition only manual annotation is employed, automatic annotation will add a lot more to tag the attributes along with the image. Automatic annotation supports more the end user, who often uploads the images over web. The end user is made free to from declaration of attributes while upload through automatic annotation.

In this model the search is dependent over the attributes being specified or declared by the user. The user must declare relevant attributes for the image being uploaded. Any erroneous attribute being declared can bring out a duplicate image irrelevant to the query given in the search. Thus produce a non semantic search. 
In this model the end user has to instruct to declare relevant attributes for their image being uploaded.

\section{REFERENCES}

[1] L. Brin, S. Page, "The anatomy of a large-scale Hyper textual Web search engine," Computer Networks and ISDN Systems, vol. 30, pp. 107-117, 1998.

[2] Berners-Lee, T., Hendler, J., and Lassila, O., 2001, "The Semantic Web", Scientific American, 284: 34-43.

[3] W3C Semantic Web Activity, 1994-2006, http://www.w3.org/2001/sw/.

[4] Gerber A.J., Van der Merwe A.J. and Barnard A., "Towards a Semantic Web Layered Architecture", In Proceedings of the International Conference on Software Engineering (IASTED SE2007), February 13-15, 2007, Innsbruck, Austria, ISBN: 978-0-88986-641-6.

[5] Liyang Yu, "Introduction to the Semantic Web and Semantic Web Services", Chapman \& Hall/CRC, Taylor \& Francis Group publication.

[6] I. Horrocks and J. Hendler, "The Semantic Web", in proceedings of First International Semantic Web Conference, Sardinia, Italy, June 9-12, 2002.

[7] S. Decker, S. Melnik, F. van Harmelen, D. Fensel, M. Klein, J. Broekstra, M. Erdmann and I. Horrocks, "The Semantic Web: The Roles of XML and RDF", IEEE Internet Computing, Vol. 4(5), pp. 63-74, Sept/Oct 2000.

[8] Resource Description Framework (RDF), http://www.w3.org/RDF/

[9] Lassila \& Swick, "Resource Description Framework (RDF) Model and Syntax Specification", O. Lassila and R. Swick (eds.): World Wide Web Consortium Recommendation, 1999.

[10] Manola et al., "RDF Primer", F. Manola, E. Miller (eds.): W3C Proposed Recommendation December, 2003,

[11] Atanas Kiryakov, Borislav Popov, Ivan Terziev, Dimitar Manov, Damyan Ognyanoff, "Semantic annotation, indexing, and retrieval" J. Web Sem. 2(1): 49-79 (2004).

[12] Semantic Annotation, http://www.ontotext.com/kim/ semanticannotation.html

[13] K.S. Candan, H. Liu, and R. Suvarna, "Resource Description Framework: Metadata and Its Applications", ACM SIGKDD Explorations, July 2001.

[14] Douglas Heflin, J., "Towards the Semantic Web: Knowledge Representation in a Dynamic, Distributed Environment", PhD Thesis, 2001.

[15] L.Hollink, A.Th.Schreiber, J.Wielemaker, B.Wielinga. "Semantic Annotation of Image Collections", In proceedings of the KCAP'03 Workshop on Knowledge Capture and Semantic Annotation, Florida, October 2003.

[16] Oge Marques and Nitish Barman, "Semi-automatic semantic annotation of Images using machine learning techniques", International Semantic Web Conference (ISWC) 2003, October 20-23, 2003, Sanibel Island, FL.

[17] Osman, T., Thakker, D., Schaefer, G., Leroy, M., and Fournier, A., "Semantic Annotation and Retrieval of Image Collections", In the proceedings of the 21st European Conference on Modelling and Simulation,

[18] Elahi, N., Karlsen, R., Akselsen, S. "A Context Centric Approach for Semantic Image Annotation and Retrieval" Future Computing, Service Computation, Cognitive, Adaptive, Content, Patterns, 2009. COMPUTATION WORLD'09.

[19] Rani Meenu, Batra Shalini, "Querying RDF model using various query engines". ME Thesis.

[20] Shelley Powers, "Practical RDF" O’Reilly, 2003 ISBN: 0596-00263-7.

[21] F. Manola, E. Miller, "RDF Primer: W3C Proposed Recommendation",

[22] RDF/XML Syntax Specification (Revised), http://www.w3.org/TR/REC-rdfsyntax/.

[23] Chao-Qing Lv, Takashi Kobayashi, Kiyoshi Agusa, Wu Kun, Zhu Qing, "Image Semantic Search Engine", Proceeding Intl Workshop on Database Technology and Applications EDBTA2009 Epp.156-159, Wuhan China, 2009.

[24] Erik Wilde, Katrina Rhoads Lindholm, "XML Foundations", September, 2006, http://dret.net/ netdret/docs/wilde-elpub2006-xml.pdf. 51

[25] Thomas Baker, Fraunhofer-Gesellschaft, "Introduction to Semantic Web", CASHMERE-int Workshop Gottingen, Germany 2005-02-28.

[26] Grau B.C., "A Possible Simplification of the Semantic Web Architecture" In WWW '04: Proceedings of the 13th international conference on World Wide Web, pages 704713. ACM Press, New York, NY, USA, 2004. ISBN 158113-844-X.doi: http://doi.acm.org/10.1145/988672.988769.

[27] Berners-Lee T. Semantic Web - XML2000. W3C Website, 2000. URL http://www.w3.org/2000/ Talks/1206-xml2ktbl/slide 10-0.html.

[28] K.S. Candan, H. Liu, and R. Suvarna, "Resource Description Framework: Metadata and Its Applications", ACM SIGKDD Explorations, July 2001.

[29] Enhancing image search accuracy using semantic annotation by Satyavir Singh. 